\title{
New Insight of Molecular Interaction, Crystallization and Phase Separation in Higher Performance Small Molecular Solar Cells Via Solvent Vapor Annealing
}

Ke Gao ${ }^{a b \Downarrow l}$ Wanyuan Deng, ${ }^{b \| l}$ Liangang Xiao, ${ }^{b \| l}$ Qin Hu, ${ }^{d}$ Yuanyuan Kan, ${ }^{b}$ Xuebin Chen, ${ }^{b}$ Cheng Wang, ${ }^{e}$ Fei Huang, ${ }^{b}$ Junbiao Peng, ${ }^{b}$ Hongbin Wu, ${ }^{b, *}$ Xiaobin Peng ${ }^{b, *}$ Yong Cao, ${ }^{b}$ Thomas P. Russell, ${ }^{c, d}$ * and Feng Liu, ${ }^{a, d *}$

${ }^{a}$ Department of Physics and Astronomy, Shanghai Jiaotong University, Shanghai, 200240, China. Email: fengliu82@sjtu.edu.cn

${ }^{\mathrm{b}}$ Institute of Polymer Optoelectronic Materials and Devices, State Key Laboratory of Luminescent Materials and Devices, South China University of Technology, 381 Wushan Road, Guangzhou 510640, China.

Email: chxbpeng@scut.edu.cn, hbwu@scut.edu.cn

${ }^{c}$ Polymer Science and Engineering Department, University of Massachusetts, Amherst, MA 01003, United States.

Email: russell@mail.pse.umass.edu

${ }^{\mathrm{d}}$ Materials Sciences Division, Lawrence Berkeley National Lab, Berkeley, CA, 94720, United States.

eAdvanced Light Source, Lawrence Berkeley National Lab, Berkeley, CA, 94720, United States.

(C) 2016. This manuscript version is made available under the Elsevier user license http://www.elsevier.com/open-access/userlicense/1.0/ 
Abstract: Solvent vapor annealing (SVA) studies on the morphology and performance of a porphyrin-based deep-absorption organic solar cells consisting of a strongly segregated bulk heterojunction (BHJ) blend, are presented. It is seen that the solvent vapor annealing of a well-mixed BHJ blends induces molecular motion, leading to a phase separated morphology governed by a spinodal decomposition mechanism. The earlier stage of solvent vapor swelling $(<10 \mathrm{~s})$ led to an obvious phase separation but not device performance. The device performance showed a dramatic increase in short circuit current and fill factor between 15-20s of SVA. Thus, phase purity is a critical parameter in determining the performance of this binary blend. SVA on a thermally annealed BHJ thin film showed two distinctive processes, a crystal dissolution and a recrystallization, accompanied by phase mixing and then phase separation. The final morphology of SVA films that were initially thermally annealed showed a reduced length scale of phase separation, in comparison to SVA on as-cast films. Thus preformed donor crystallites appear to lock-in the morphology, even in a small molecule blend setting. The best performing device was obtained by a slight SVA (10s) of films that were initially thermally annealed, reaching a power conversion efficiency of $8.48 \%$. This suggests that the localized morphological optimization and domain size reduction are most important factors in dictating organic photovoltaic device efficiencies.

Keywords: organic solr cells, solvent vapor annealing, non-equilibrium morphology, physical processes

\section{Introduction}

In recent years, photon active small-molecule donor and acceptor materials have advanced significantly in organic photovoltaics. [1-6] Unlike their polymer analogues, 
small molecules can be readily synthesized, be purified with better and controlled quality, and have a molecular weight distribution of unity. In addition, small molecules have a lower viscosity and better structural order that can be used to manipulate the active layer morphology and performances. [7] Solar cells using small molecule donor materials have achieved power conversion efficiencies (PCEs) of more than 10\%; [1,2] and small molecule acceptors are now comparable to fullerene derivatives. [1,2,8] From the laboratory research setting to the manufacturing line, the morphological parameters for small-molecule-based active layer blends must be optimized to obtain the best performance. ${ }^{9,10}$ In many cases, small molecule donors and fullerene-based acceptors can be mixed and the rapid crystallization during solution casting gives rise to high quality BHJ morphologies reflected in good device performance. [11-14] Often, thermal annealing (TA) at relatively low temperatures or solvent vapor annealing (SVA) is done to enhance the crystallization of the small molecules and improve the morphology. $[1,3,15-20]$ Solvent vapor annealing has been proven to be a very effective route to control the morphology, due to the reduced viscosity, the high degree of crystallinity of the small molecule and the fast kinetics. In SVA process, the penetration of solvent molecules swells the BHJ layer, decreasing the effective glass transition temperature and markedly enhancing the molecular mobility. [18] Both crystallization and phase separation can be accelerated, driving the evolution of the morphology. Consequently, the choice of solvent(s) and the duration of the swelling are critical.

Conjugated molecules are hierarchical in molecular structure, facilitated by an oligomeric backbone and aliphatic side chains. The intermolecular interactions can be quite complex, which would affect their self-assembly and electronic properties. In this 
report, we focus on the morphological manipulation of a porphyrin based low energy loss, high performance donor small molecule DPPEZnP-TBO (5,15-Bis(2,5-bis-(2-ethylhexyl)-3,6-di-thienyl-2-yl-2,5-dihydro-pyrrolo[3,4-c]pyrrole-1,4-dione-5'-yl-ethynyl)10,20-bis(5-(2-butyloctyl)-thienyl)-porphyrin zinc(II)). [21,22] The chemical structure is shown in Scheme 1. DPPEZnP-TBO has a porphyrin center and two DPP arms. The aromatic plane of DPPEZnP-TBO is quite large, thus $\pi-\pi$ stacking can be complex. DPPEZnP-TBO has been shown to form either $\mathrm{J}$ or $\mathrm{H}$ aggregates, with the thin film crystalline order is low in the as-cast BHJ blends. [22] It was also noted that its mixing with a PCBM acceptor could be controlled by adding a pyridine additive that formed an intermediate mixing state and could be further manipulated to generate a new morphology. [21] Moreover, thermal annealing could be used to induce de-mixing of the BHJ blends. [21] The current study focuses on the SVA treatment of DPPEZnP-TBO BHJ thin films to control the morphology and on correlating morphological details with device characteristics. 


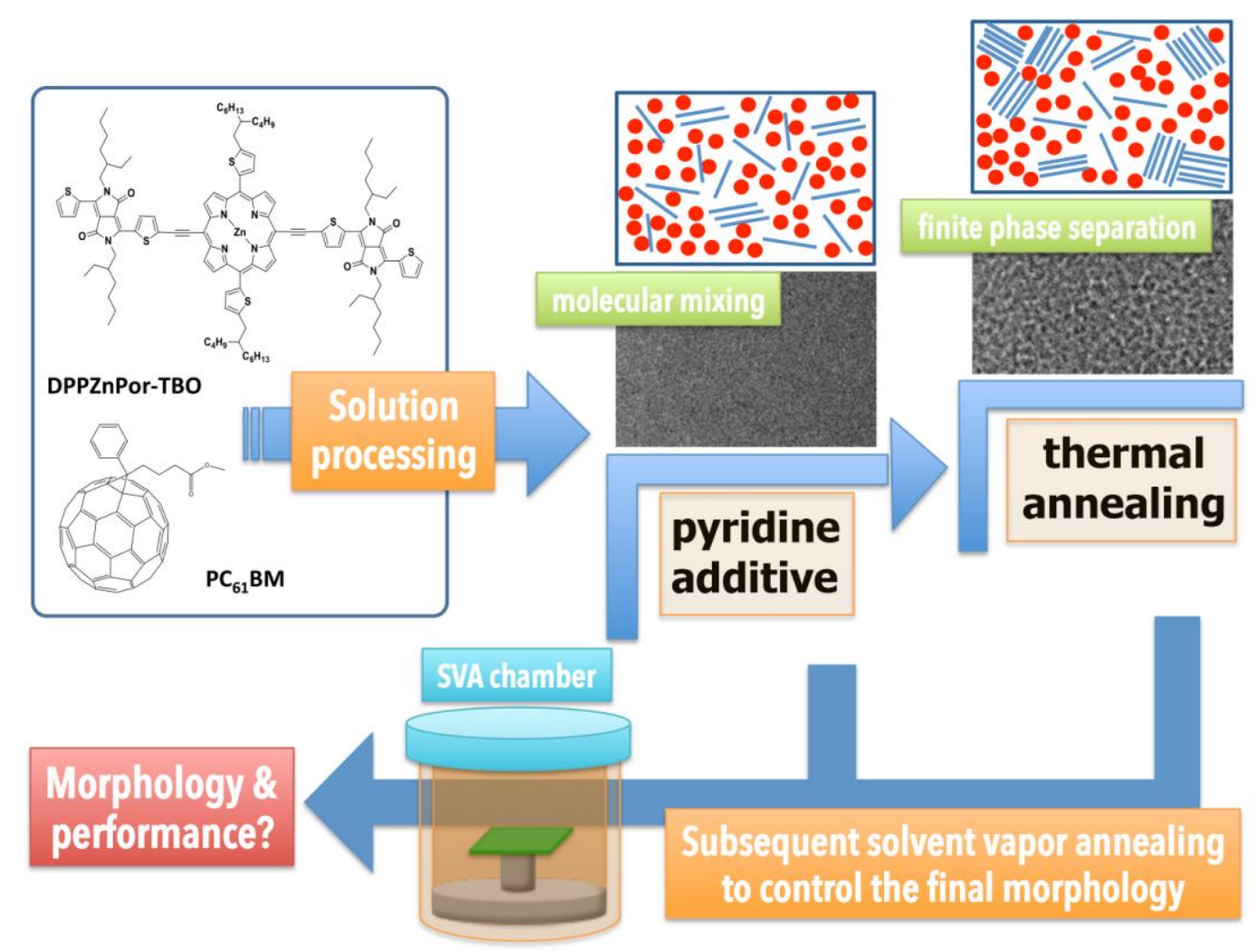

Scheme 1. Solvent vapor annealing experiment design.

The scope and experimental design of the studies are shown in Scheme 1. BHJ thin films were first solution cast from chlorobenzene using pyridine as an additive to obtain homogenously mixed films. Films were then thermally annealed at $100^{\circ} \mathrm{C}$ for $10 \mathrm{~min}$. Both the as-cast and thermally annealed films were solvent vapor annealed (SVA) in tetrahydrofuran (THF) vapor for different periods of time to manipulate morphology and device performance. The as-cast films rapidly phase separated state with a characteristic length scale of $\sim 78 \mathrm{~nm}$. When the thermally annealed samples were subjected to SVA, a dissolution of DPPEZnP-TBO crystals occurred initially, followed by a phase separation with a characteristic length scale that was much smaller than the SVA-as-cast films, leading to markedly different device performances. The physical processes of the two SVA treatments are thus quite different and related to the local chemical environments. 
These details as well as electronic properties of BHJ thin films were thoroughly characterized and structure-property relationship was proposed.

\section{Results and discussions}

\subsection{Optical properties and aggregation behavior}
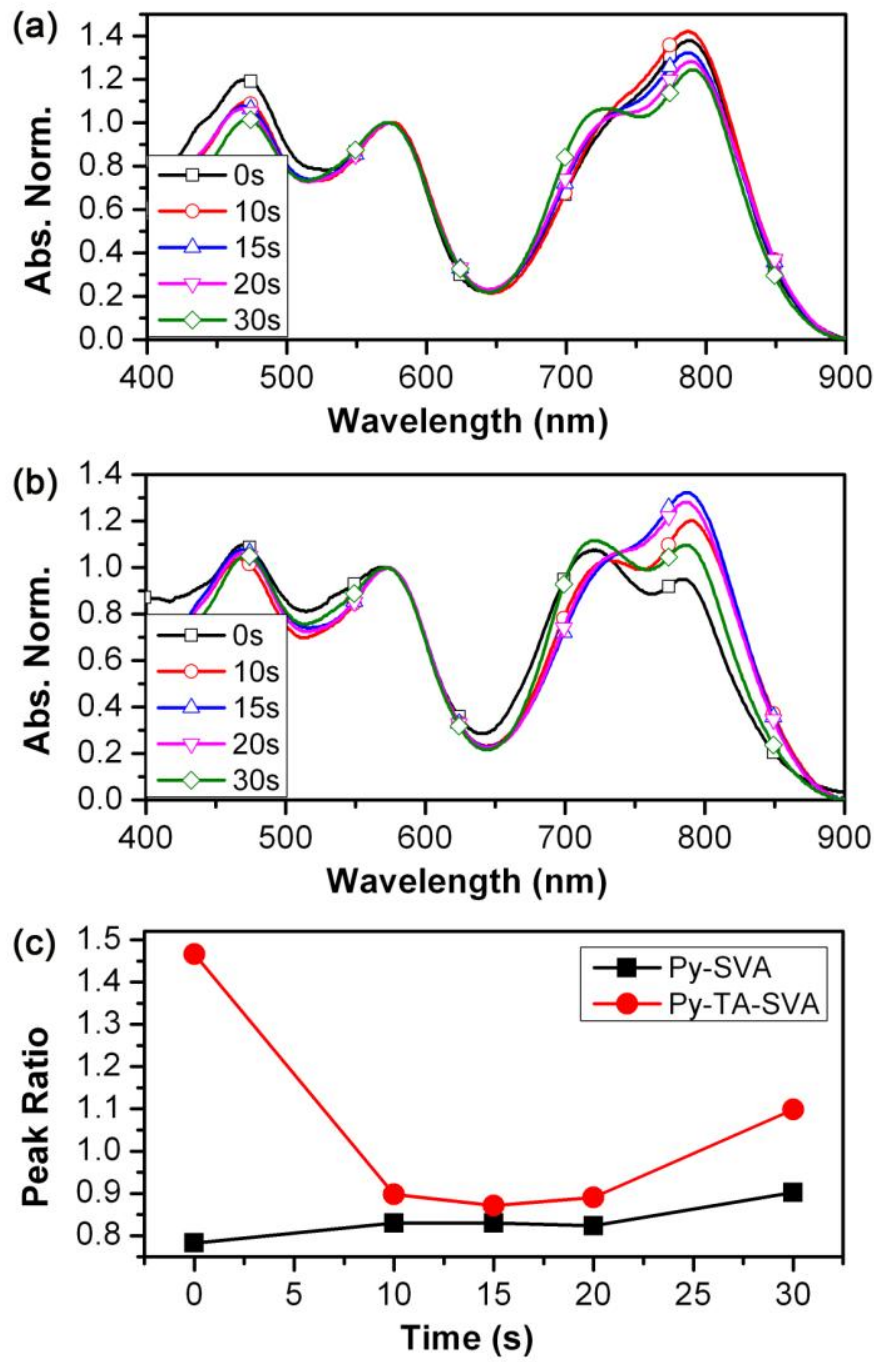

Figure 1. Normalized UV-vis absorption spectra of DPPZnPor-TBO: PCBM blended films using Py-SVA (a) and Py-TA-SVA (b) processing methods; (c) The ratio of splitted $\sim 730 \mathrm{~nm}$ to $\sim 790 \mathrm{~nm}$ peak heights. 
The UV-vis absorption spectra of the SVA on as-cast and thermally annealed BHJ thin films with a 1:1.2 (donor: acceptor) weight ratio are shown in Figure 1. Tetrahydrofuran was used as the annealing solvent, since it is a good solvent for DPPZnPor-TBO and has a moderate to high vapor pressure at atmosphere conditions. The blend films processed with pyridine had three major absorption peaks at 469,574 , and $790 \mathrm{~nm}$ (Figure 1a). A small peak or shoulder was observed at $\sim 730 \mathrm{~nm}$. The $790 \mathrm{~nm}$ peak is associated with $\mathrm{J}$-aggregates of porphyrin molecules, while the $730 \mathrm{~nm}$ peak arises from H-aggregates. With SVA the absorption of the high wavelength region remained essentially unchanged with a slightly reduced $790 \mathrm{~nm}$ peak intensity during the first 20s. After 30s SVA the intensity of the shoulder increased significantly to form a well-developed peak. Thus, the molecular packing shifted from $\mathrm{J}$ - to $\mathrm{H}$-aggregates, by a sliding of the intermolecular $\pi-\pi$ stacking to adopt different quadruple interactions, as demonstrated by Bredas and coworkers, [23] which could lead to significant changes in the electronic properties. Solution cast film, using pyridine as an additive and then thermally annealed, are summarized in Figure 1b. These samples showed a strong absorption at $720 \mathrm{~nm}$, with a minor absorption at $784 \mathrm{~nm}$. After 10s of SVA, the two absorptions red shifted to $\sim 730 \mathrm{~nm}$ and $\sim 790 \mathrm{~nm}$, respectively, with the $790 \mathrm{~nm}$ absorption dominating. Consequently, the molecular packing changed from $\mathrm{H}-$ to $\mathrm{J}$ aggregates. After 20s of SVA the intensity of the absorption band at $790 \mathrm{~nm}$ continued to increase while the intensity of the absorption at $730 \mathrm{~nm}$ decreased. However, after $\sim 30 \mathrm{~s}$ of SVA, the molecular packing reverted to an $\mathrm{H}$ - aggregate. The peak ratios of the absorptions characteristics of the $\mathrm{H}$ - to J-aggregates were estimated by fitting the profiles to two Gaussian functions. The results are summarized in Figure 1c. It is evident that 
there were different kinetic pathways for the two SVA procedures. In as-cast and SVA thin films, the molecular packing shifted towards an H-aggregate, which was initially slow but reached a plateau after 10-20s, and then began to evolve more rapidly. However, even at the end of SVA ( 30s), the concentration of H-aggregates was lower than the concentration of J-aggregates, as evidenced by the absorption profiles. For TA and SVA samples, the molecular packing rapidly shifted from $\mathrm{H}$ - to J-aggregates, plateaued, and then began to shift back to an H-aggregated state. The difference in these two indicates that two different states of phase separation existed. Therefore, the TA-SVA experiments can be separated into two sub-processes, which will be further analyzed. The complicated $\mathrm{H}$ and $\mathrm{J}$ aggregation change can arise from the complex molecule structure. The porphyrin molecule is fairly planar. The acetylene connected DPP moieties extends the conjugation. The aromatic plane is quite large. The intermolecular interactions can thus be quite complex. There could be well-ordered cofacial stackings or fractional stackings with adjacent molecules, leading to reduced structural order. When the solvent molecules get into the films, the molecular mobility became very high. Conjugated molecules will reorganize and order, which changes the spatial overlap between the stacking pi orbitals.[23] Thus the transfer integral and UV absorption change, with the results seen from our absorption investigation. 


\subsection{Device performances}

Table 1. Summary of device parameters of organic solar cells processed with different conditions under simulated AM 1.5G irradiation $(100 \mathrm{~mW} \mathrm{~cm} 2)$.

\begin{tabular}{|c|c|c|c|c|c|c|c|}
\hline $\begin{array}{l}\text { Condition } \\
\mathrm{s}^{\mathrm{a}}\end{array}$ & $\begin{array}{c}\text { SVA } \\
\text { time } \\
(\mathrm{s})\end{array}$ & $\begin{array}{c}\mathbf{J}_{\mathrm{sc}} \\
(\mathbf{m A} \mathbf{m} / \mathbf{c m} \\
\mathbf{2} \\
\end{array}$ & $\begin{array}{l}\text { Voc } \\
(\mathrm{V})\end{array}$ & $\begin{array}{l}\text { FF } \\
(\%)\end{array}$ & $\begin{array}{l}\text { PCE } \\
(\%)\end{array}$ & $\begin{array}{c}\mathbf{P C E}^{\mathbf{b}} \\
(\%)\end{array}$ & $\begin{array}{c}\mathbf{J}_{\mathrm{sc}, \mathrm{cal}}{ }^{\mathrm{c}} \\
(\mathbf{m A} / \mathbf{c} \\
\left.\mathbf{m}^{2}\right) \\
\end{array}$ \\
\hline \multirow{6}{*}{$\mathbf{P y}$} & 0 & 9.0 & 0.87 & 32 & 2.5 & 2.4 & 8.7 \\
\hline & 10 & 10.2 & 0.86 & 34 & 3.0 & 2.9 & 9.8 \\
\hline & 15 & 11.5 & 0.80 & 41 & 3.8 & 3.6 & 11.2 \\
\hline & 18 & 15.2 & 0.78 & 56 & 6.6 & 6.5 & 14.6 \\
\hline & 20 & 16.5 & 0.77 & 62 & 7.9 & 7.8 & 15.7 \\
\hline & 30 & 15.2 & 0.71 & 70 & 7.6 & 7.4 & 14.6 \\
\hline \multirow{5}{*}{ Py+TA } & 0 & 17.4 & 0.79 & 57 & 7.8 & 7.7 & 16.9 \\
\hline & 10 & 17.2 & 0.78 & 63 & 8.5 & 8.3 & 16.7 \\
\hline & 15 & 16.8 & 0.77 & 64 & 8.3 & 8.2 & 16.4 \\
\hline & 20 & 16.8 & 0.72 & 67 & 8.1 & 8.0 & 16.2 \\
\hline & 30 & 16.4 & 0.71 & 69 & 8.0 & 7.9 & 15.9 \\
\hline
\end{tabular}

${ }^{\mathrm{a}} \mathrm{Py}$ : chlorobenzene $+0.4 \mathrm{v} \%$ pyridine additive processing; Py+TA: chlorobenzene $+0.4 \mathrm{v} \%$ pyridine additive processing, then thermal annealing; ${ }^{\mathrm{b}}$ average PCE from 25 devices; ${ }^{\mathrm{c}} \mathbf{J}_{\text {sc,cal }}$ : integrated Jses from the EQE

Solar cell devices were fabricated using a standard device structure (ITO/PDEDOT:PSS/ DPPZnPor-TBO:PCBM/PFN/Al). Two series of devices, as-cast and thermally annealed BHJ thin films, subjected to different SVA times were investigated. The optimized photovoltaic parameters are summarized in Table 1. The current density-voltage $(J-V)$ characteristics of solar cells for the as-cast-SVA and the TA-SVA series are shown in Figures $2 \mathrm{a}$ and $2 \mathrm{~b}$, respectively. For the as-cast-SVA series, as cast devices showed a PCE of $2.5 \%$, with open circuit voltage $\left(\mathrm{V}_{\mathrm{oc}}\right)$ of $0.87 \mathrm{~V}, \mathrm{~J}_{\mathrm{sc}}$ of $9.0 \mathrm{~mA} \mathrm{~cm}^{-2}$, and $\mathrm{FF}$ of $32 \%$. These are typical features of BHJ blends that showed good mixing and with no pathways for electron and hole transport. [24,25] After 10s of annealing in THF vapor, immediate improvements in the $\mathrm{J}_{\mathrm{sc}}=10.2 \mathrm{~mA} \mathrm{~cm}$ and PCE $=$ $3.0 \%$ were observed. SVA for $15 \mathrm{~s}$ increased the $\mathrm{J}_{\mathrm{sc}}=11.5 \mathrm{~mA} \mathrm{~cm}{ }^{-2}$, and $\mathbf{F F}=41$, with a 
loss in $\mathrm{V}_{\mathrm{oc}}$ to $0.80 \mathrm{~V}$, giving a PCE of $3.8 \%$. After a $20 \mathrm{~s}$ SVA, the $\mathrm{J}_{\mathrm{sc}}$ of the device increased to $16.5 \mathrm{~mA} \mathrm{~cm}^{-2}$, the fill factor increased to 62 , and the PCE was $7.9 \%$. With further SVA, though the FF increased to $\mathbf{7 0}$, both the $\mathrm{V}_{\mathrm{oc}}$ and $\mathrm{J}_{\mathrm{sc}}$ decreased, with a PCE of $7.6 \%$ being recorded. Thus in this series of experiment, the first 10s of SVA of the solvent annealed samples brought little change. After 18s SVA, the PCE increased to 6.7\%. After 20s SVA, all the parameter increased further, yielding the best performances in this series. Further, SVA led to a deterioration in the device performance. As a whole, the PCE continuously increased from 0 s to 30 s, corresponding to the increase in the percentage of $\mathrm{H}$ aggregates which is beneficial for charge transfer. Consequently, the Jsc and PCE increased.

The device processed with pyridine and then thermally annealed had a PCE of 7.8\%, with a $\mathrm{V}_{\mathrm{oc}}$ of $0.79 \mathrm{~V}$, a $\mathrm{J}_{\mathrm{sc}}$ of $17.4 \mathrm{~mA} \mathrm{~cm}^{-2}$, and a FF of 57 . After 10s of SVA the device had a best PCE of $8.5 \%$, arising from the improved FF. Further SVA led to a slight decrease in $\mathrm{V}_{\mathrm{oc}}$ and $\mathrm{J}_{\mathrm{sc}}$, with the PCE decreasing to $8.3 \%$. The device FF continued to increase with prolonged SVA, reaching 69 after 30s of SVA, indicating that the degree of phase separation increased. It was shown previously that BHJ thin films processed from pyridine additive and thermal annealing would lead to a crystallization of DPPZnPorTBO and a well-defined small length scale phase separation. [21,22] SVA led to H- to Jaggregation and then, with prolonged annealing back to H-aggregation. The crystallites originally formed by thermal annealing were dissolved and recrystallized, associated with a localized molecular movement and reorganization. Understanding the localized chemical environment, and the morphological parameters are the key to understanding the device performances. From 0s to 30s, the PCE decreased and then increased, which 
countered the change in the amount of $\mathrm{H}$ aggregates. This suggests that the changes of the molecular aggregation are not the major source of the changes of the PCE in this series and the morphology dominates.

As shown in Figure 2c, the as-cast and SVA thin films showed a progressive enhancement in EQE up to 20s of annealing, and then began to decrease. In TA-SVA samples, the original EQE curve was quite high. Subsequent SVA, in fact, reduced the $\mathrm{EQE}$, giving rise to the reduced $\mathrm{J}_{\mathrm{sc}}$ value. More detailed device parameter statistics are shown in Figures 2e-h. The comparison of these values reflects the distinctive features of the two processes. In both cases, a transition is seen between 10-20s of SVA, which is related to a change in the morphology. This correlates with the spectroscopy studies where a plateau was seen in the amount of J-aggregates that formed. For the Py-SVA samples, 10-20s of SVA corresponds to the time when phase separation was optimized and a change in the electronic structure of the film, as evidenced by the $\mathrm{V}_{\mathrm{oc}}$ and concentration of J-aggregates, was observed. 

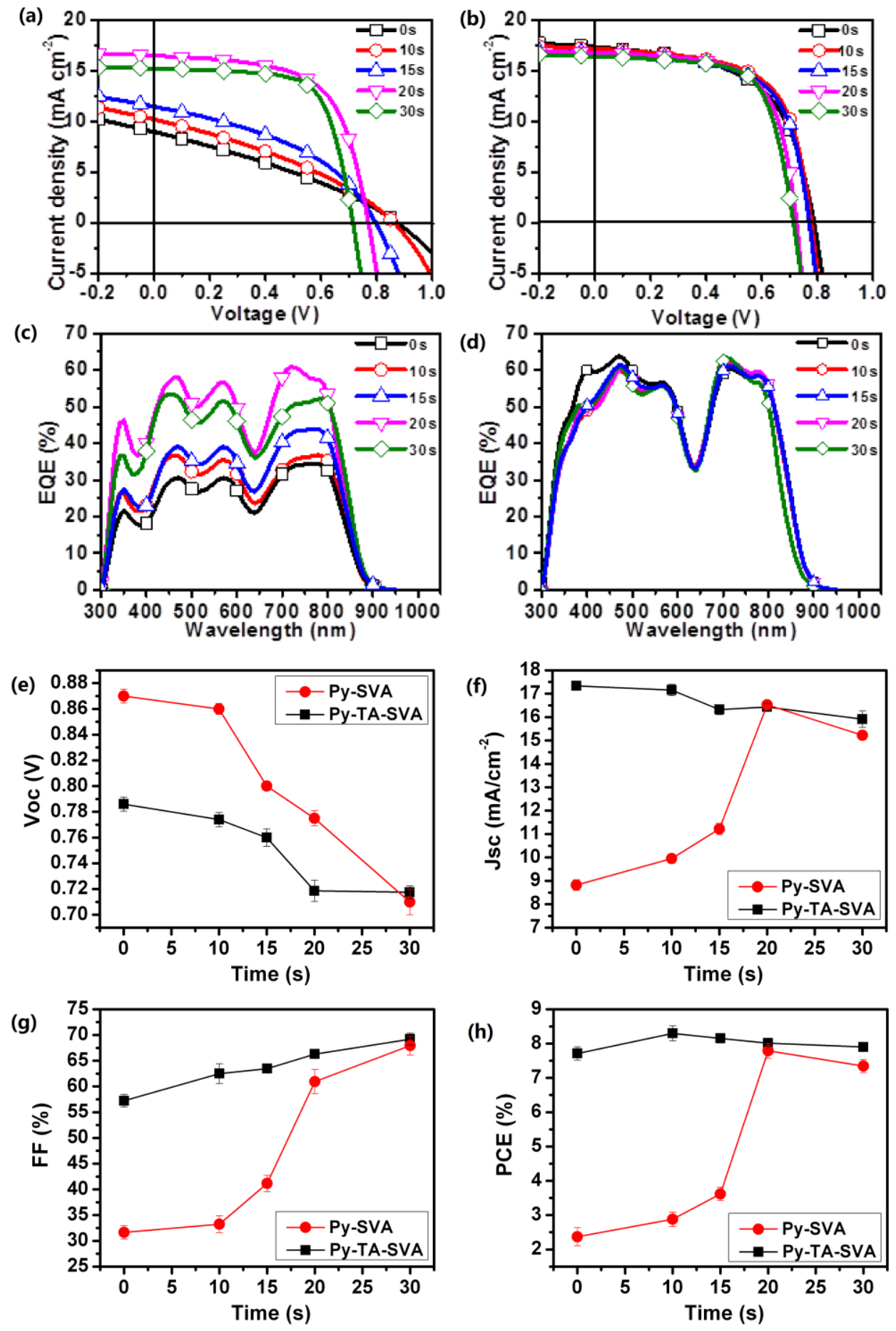

Figure 2. (a) $J-V$ curves of solar cells processed using Py-SVA procedure; ( b) $J-V$ curves of solar cells processed using Py-TA-SVA procedure; (c) and (d) the corresponding EQE of solar cells for (a) and (b); (e)-(h) device parameter statistics. 


\subsection{Electrical property characterizations}

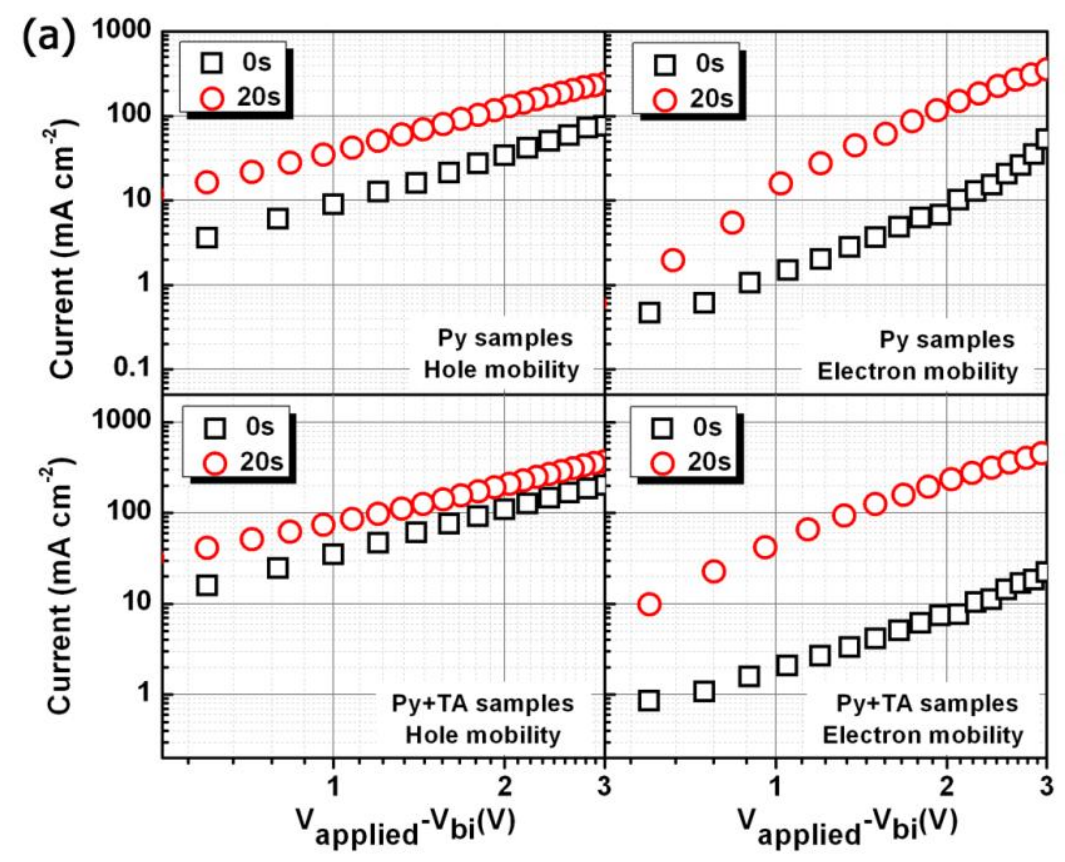

(b)

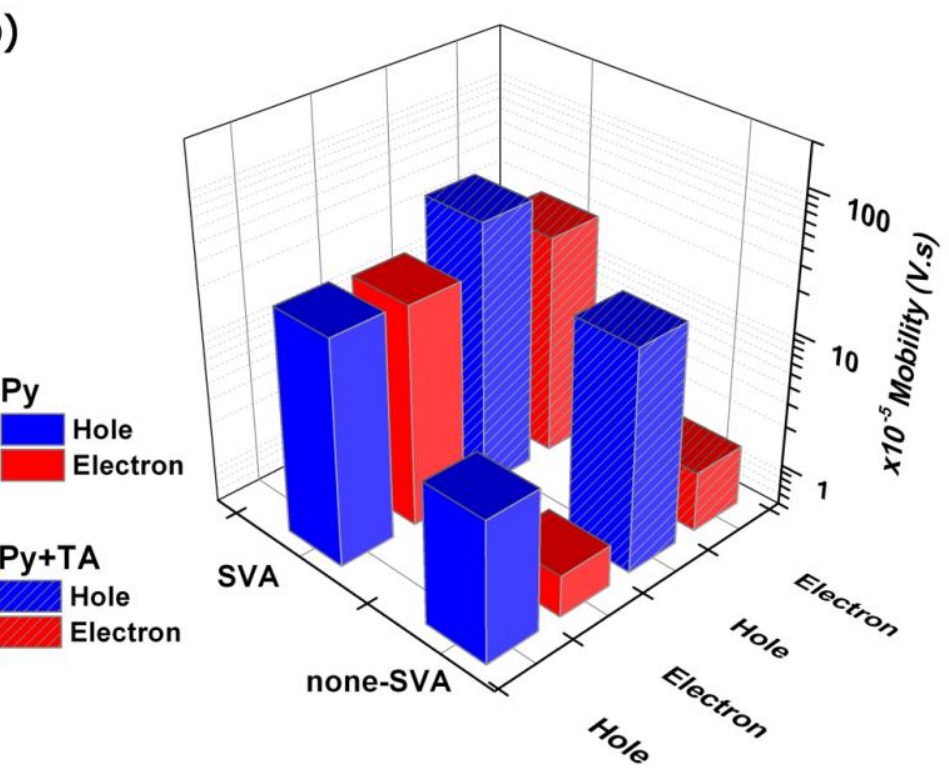

Figure 3. (a) Single carrier device SCLC measurement for DPPZnPor-TBO: PCBM blended films using Py-SVA and Py-TA-SVA processing methods; (b) comparison of carrier mobility by different processing methods.

The electronic properties of the BHJ thin films were further characterized to understand the device performance. Shown in Figure 3 are the single carrier mobility 
measurements of Py-SVA and Py-TA-SVA thin films using space charge limited current (SCLC) method. [24] The initial and 20s SVA samples are compared. As shown, for the initial films (0s SVA), the electron mobilities in both cases were $\sim 1 \times 10^{-5} \mathrm{~cm}^{2} \mathrm{~V}^{-1} \mathrm{~s}^{-1}$, which are much lower than the hole-mobilities. The hole mobility for the Py-SVA case was $\sim 5 \times 10^{-5} \mathrm{~cm}^{2} \mathrm{~V}^{-1} \mathrm{~s}^{-1}$, and this value was $\sim 2 \times 10^{-4} \mathrm{~cm}^{2} \mathrm{~V}^{-1} \mathrm{~s}^{-1}$ in thermally annealed sample. The initial cast film from pyridine additive processing was less crystalline and the donor and acceptor mixed well. Thermal annealing significantly increased the hole mobility, due to the enhanced crystallization of the porphyrin molecule; electron mobility, though, was only slightly enhanced, which arises from the lack of reorganization of PCBM, since the annealing temperature, $100{ }^{\circ} \mathrm{C}$, was much lower than its glass transition temperature $\left(>130{ }^{\circ} \mathrm{C}\right)$. [26] When THF vapor annealing was used, the mobilities dramatically changed. As shown in Figure 3b, both electron and hole mobilities increased to a similar value. The hole mobility in Py-SVA sample reached $\sim 2.3 \times 10^{-4} \mathrm{~cm}^{2} \mathrm{~V}^{-1} \mathrm{~s}^{-1}$ and $\sim 4.6 \times 10^{-4} \mathrm{~cm}^{2} \mathrm{~V}^{-1} \mathrm{~s}^{-1}$ in the Py-TA-SVA sample. Electron mobilities in both cases were similar at $\sim 2 \times 10^{-4} \mathrm{~cm}^{2} \mathrm{~V}^{-1} \mathrm{~s}^{-1}$, since the introduction of THF enhances the mobility of the PCBM which can re-organize. Therefore, in comparison to thermal annealing, SVA is much more effective in manipulating the morphology. 
(a)

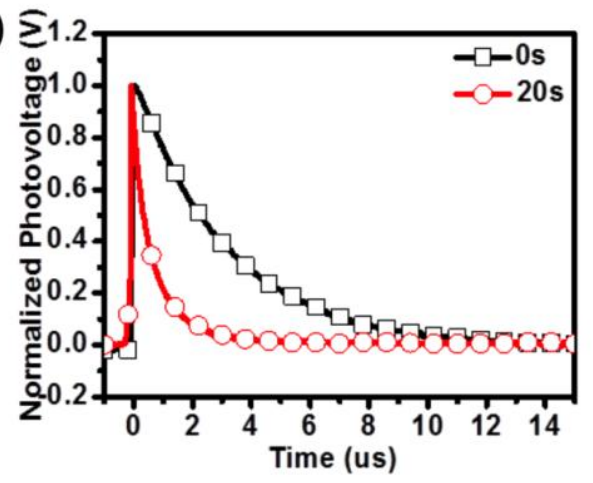

(c)

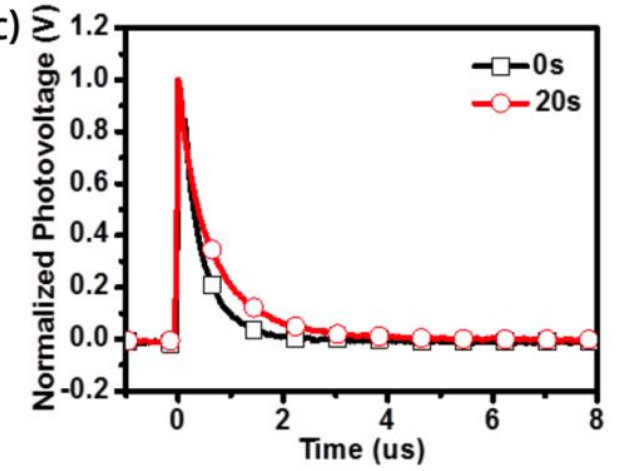

(b)

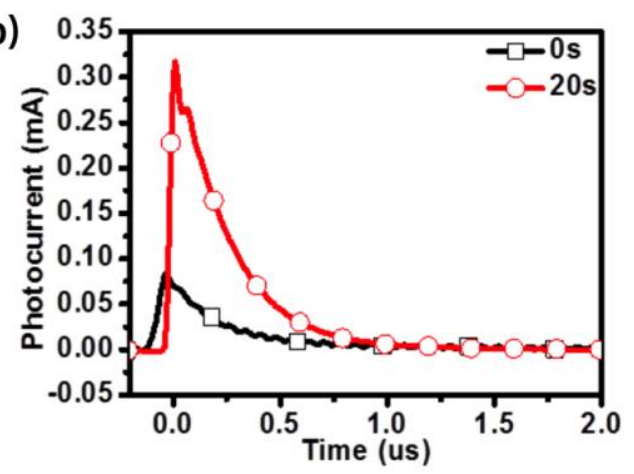

(d)

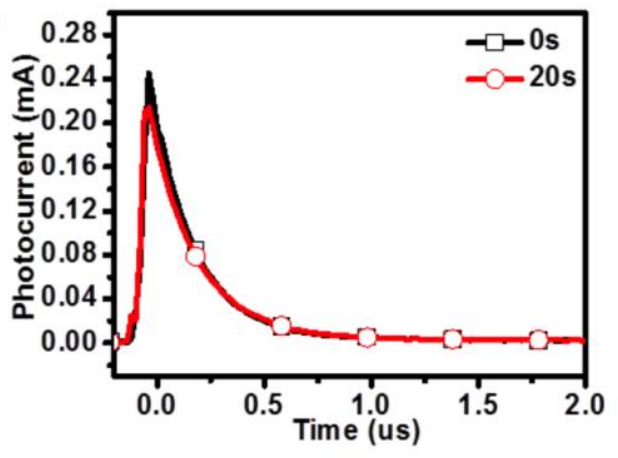

Figure 4. (a) Transient photovoltage and (b) Transient photocurrent for OPV devices processed using Py-SVA procedure; (c) Transient photovoltage and (d) Transient photocurrent for OPV devices processed using Py-TA-SVA procedure.

The charge extraction and recombination dynamics were studied by transient photovoltage (TPV) and transient photocurrent (TPC) measurements. [27-29] Shown in Figures $4 \mathrm{a}$ and $4 \mathrm{~b}$ are the results for the Py-SVA samples and Figures $4 \mathrm{c}$ and $4 \mathrm{~d}$ show the results for the Py-TA-SVA samples. In each case, the 0s and 20s SVA experiments were studied. For Py-SVA samples, the initial samples showed a slower TPV decay (data normalized), and thus a longer charge carrier lifetime. It is expected that under this condition the donor and acceptor are mixed, [30,31] where the donor-acceptor could form an "alloy" to generate a new electronic state that functions as "acceptor". The newly formed "acceptor-alloy" would have a shallower lowest unoccupied molecular orbital (LUMO), which then aligned with highest occupied molecular orbital (HOMO) of the 
porphyrin donor, to give a larger energy offset and a larger Voc. In device characterization, 0 s annealed sample gave a $\mathrm{V}_{\mathrm{oc}}$ of $0.87 \mathrm{~V}$, and $20 \mathrm{~s}$ annealed sample gave a $\mathrm{V}_{\mathrm{oc}}$ of $0.77 \mathrm{~V}$, supporting this argument. Thin films subjected to 20s SVA had a more rapid carrier decay lifetime. Thus, the charge carriers could be more effectively extracted, which resulted in a higher $\mathbf{J}_{\mathrm{sc}}$. In the TPC measurements, the photocurrent in 20s SVA device was much higher than the device with the as-cast active layer, which agreed well with the device characteristics and TPV measurements. For the Py-TA-SVA devices, TPV measurements of 0 s and 20s annealed devices showed similar decay rates. The thermally annealed as-cast devices showed a slightly more rapid decay rate, thus more efficient carrier extraction, due to the smaller length scale of the phase separated morphology and the crystallization of the porphyrin molecule. Only slight changes occurred upon SVA. It is evident that the earlier stage of SVA in the Py-SVA case results in a demixing, changing the electronic properties of the BHJ thin film, as evidenced by the drop in the $\mathrm{V}_{\mathrm{oc}}$. SVA in both cases improved the quality of phase-separated morphology and gave rise to higher and more balanced carrier mobilities. Thus, the device FF increased with prolonged SVA, which also increased the size of the phaseseparated domain, reducing the $\mathrm{J}_{\mathrm{sc}}$.
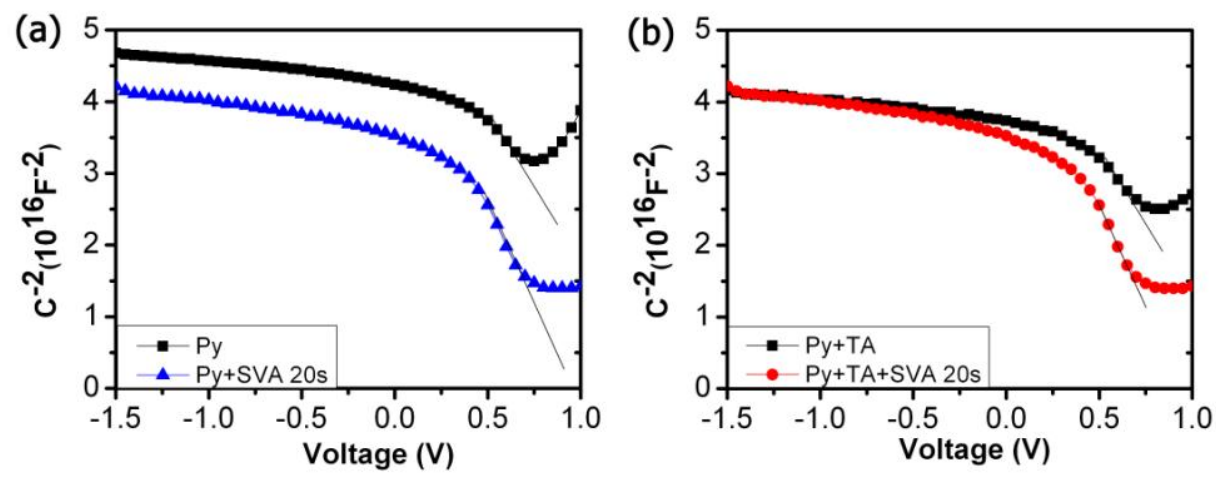

Figure 5. Capacitance-voltage measurement at $10 \mathrm{kHz}$. 
The electrical properties of BHJ thin films were further studied by capacitance measurement. Shown in Figure 5 are the capacitance-voltage $(\mathrm{C}-\mathrm{V})$ measurements at a frequency of $10 \mathrm{kHz}$. [32] According to the classical Mott-Schottky equation, the tangent line extrapolated to zero capacitance yields the intrinsic potential $\left(\mathrm{V}_{\mathrm{bi}}\right)$ of the solar cell device. [33,34] The initial Py-SVA sample gave the highest $V_{b i}$, ands a higher $V_{o c}$ would be expected. With SVA, $\mathrm{V}_{\mathrm{bi}}$ decreased, in good agreement with $\mathrm{V}_{\mathrm{oc}}$. The $\mathrm{C}-\mathrm{V}$ curves for as-cast and 20s SVA samples were quite different. The as-cast sample gave a much larger capacitance over the measured bias range. Arising, more than likely, from the well-mixed sate of the as-cast film. If DPPZnPor-TBO and PCBM mix well, the localized molecular interactions will give rise to intermolecular dipoles that will effectively increase the dielectric constant. For the Py-TA-SVA devices, the 20s SVA led to $\mathrm{V}_{\mathrm{bi}}$ drop; and the overall profiles were similar and similar to 20s Py-SVA film. The major differences were in the higher bias region, which may arise from the different states of aggregation of the PCBM that led to the electronic property change.

\subsection{Morphology characterizations}

The crystalline structure of DPPZnPor-TBO in BHJ blends was studied by grazing incidence x-ray diffraction (GIXD). [35] Shown in Figure 6 are the 2-D diffraction images and in-plane (IP)/out-of-plane (OOP) line cut profiles. As seen, the Py-SVA sample series showed a systematic growth in the crystallinity. In the as-cast sample, quite weak structural order was evident for DPPZnPor-TBO, with the diffraction profile being dominated by the diffraction from the PCBM. After 10s SVA in THF, a diffraction peak at $\sim 0.3 \mathrm{~A}^{-1}$ was observed, arising from the (100) plane of DPPZnPor-TBO. No significant azimuthal angular dependence was seen, indicating that the crystallites are randomly 
oriented. With further SVA, an enhancement of the (100) was evident, indicating an increased ordering. The crystal size along the (100) direction, as determined by a Scherrer analysis of the width of the reflection (crystal size equals to $2 \pi / f w h m$, fwhm: full-with-at-half-maximum of the diffraction peak, which is obtained by peak fitting), [36] increased from $6.0 \mathrm{~nm}$ (for the 10s SVA film) to $8.9 \mathrm{~nm}$ (for the 30s SVA annealed film). A transition in the 10-20s SVA period was noted, as evidenced by the rapid increase in the diffraction intensities. During the initial 10s time frame, the changes were minimal, which is consistent with the other measurements (spectroscopic and electronic properties) presented previously.
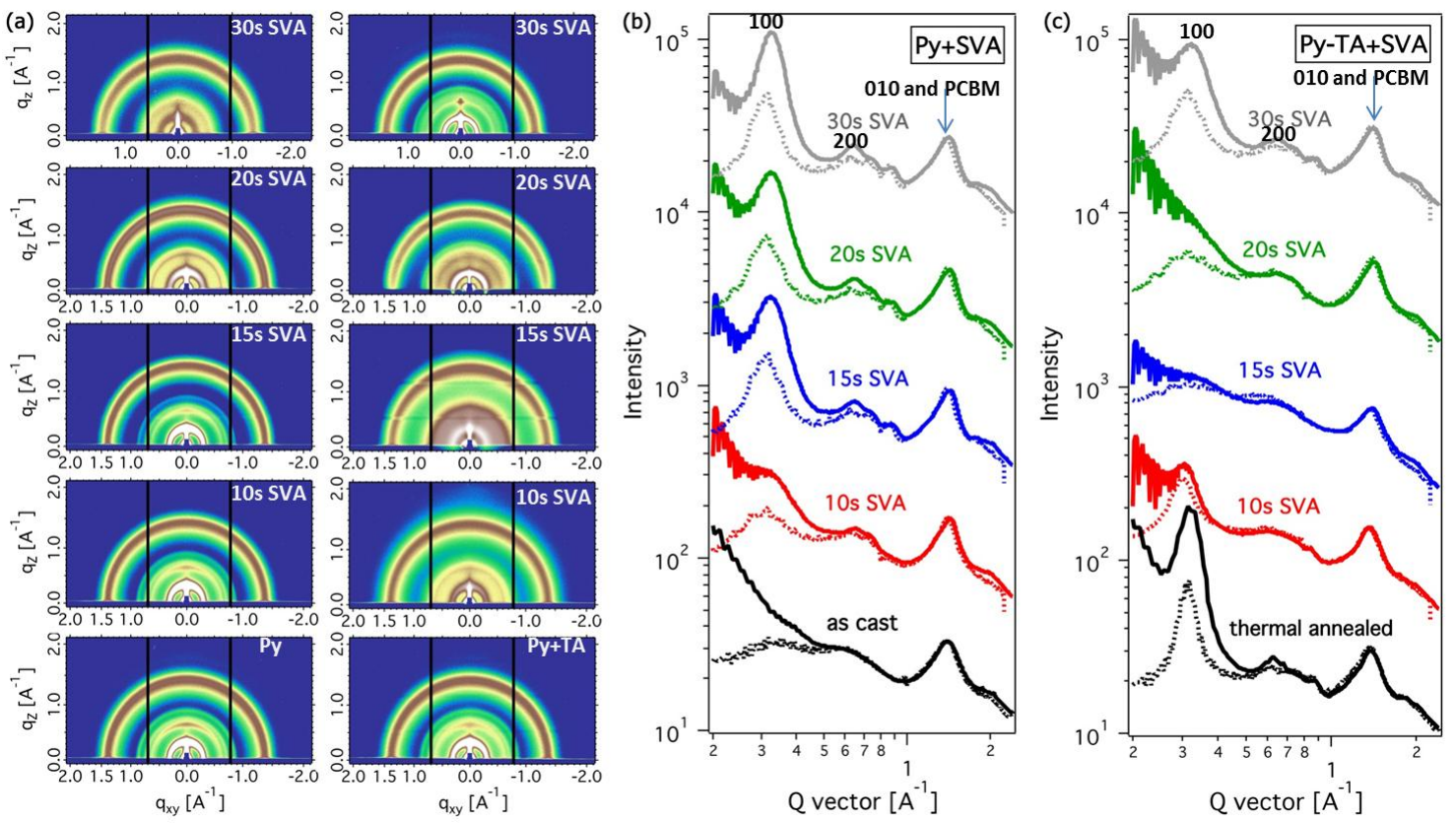

Figure 6. Grazing incidence x-ray diffraction 2-D images (a) and line cut profiles for Py-SVA (b) and Py-TA-SVA (c) processed BHJ thin films.

For the Py-TA-SVA sample series, thermal annealing at $100{ }^{\circ} \mathrm{C}$ for $\sim 10$ min led to a strong reflection of (100) plan. However, with SVA the intensities of the reflections decreased, suggesting that the THF vapor dissolves the DPPZnPor-TBO crystals. This 
continued up to $15 \mathrm{~s}$, whereupon new crystallites began to form and grow. The 30s SVA sample showed a much weaker (100) diffraction peak in comparison to the thermally annealed sample. It is interesting to note that, even though the order decreased with SVA, the devices performed quite well, with a PCE $>8 \%$. This suggests that a thermalannealing-induced phase separation plays a critical role. In the early stages of SVA, although the DPPZnPor-TBO crystalline domain began to dissolve, there was still sufficient phase separation to generate current in the device. These results suggest an interesting morphological picture. For the thermally annealed sample, a well-defined phase separation occurred due to crystallization. The annealing temperature of $100{ }^{\circ} \mathrm{C}$, is well below the $\mathrm{T}_{\mathrm{g}}$ of the PCBM and, consequently, motion of the PCBM is slow and crystallization occurs in a framework established by the glassy PCBM. Thus, in this thermal annealing induced demixing procedure, PCBM is pushed by DPPZnPor-TBO molecule, and it is envisioned that at phase separation boundaries, interfacial stress existed, that influenced the electronic status of the porphyrin molecule crystallites and BHJ thin film. In fact, thermally annealed thin film showed an $\mathrm{H}$-aggregation with the absorption peak located at $720 \mathrm{~nm}$, which is slightly shorter in comparison to the SVA induced H-aggregation. Thus the thermally induced crystal quality is less favorably packed and cross-molecule electron communication is weaker, which can be the result of internal stress induced by the interfacial constraint. [37] The subsequent SVA can dissolve these crystals and recrystallization occurs. [38] 

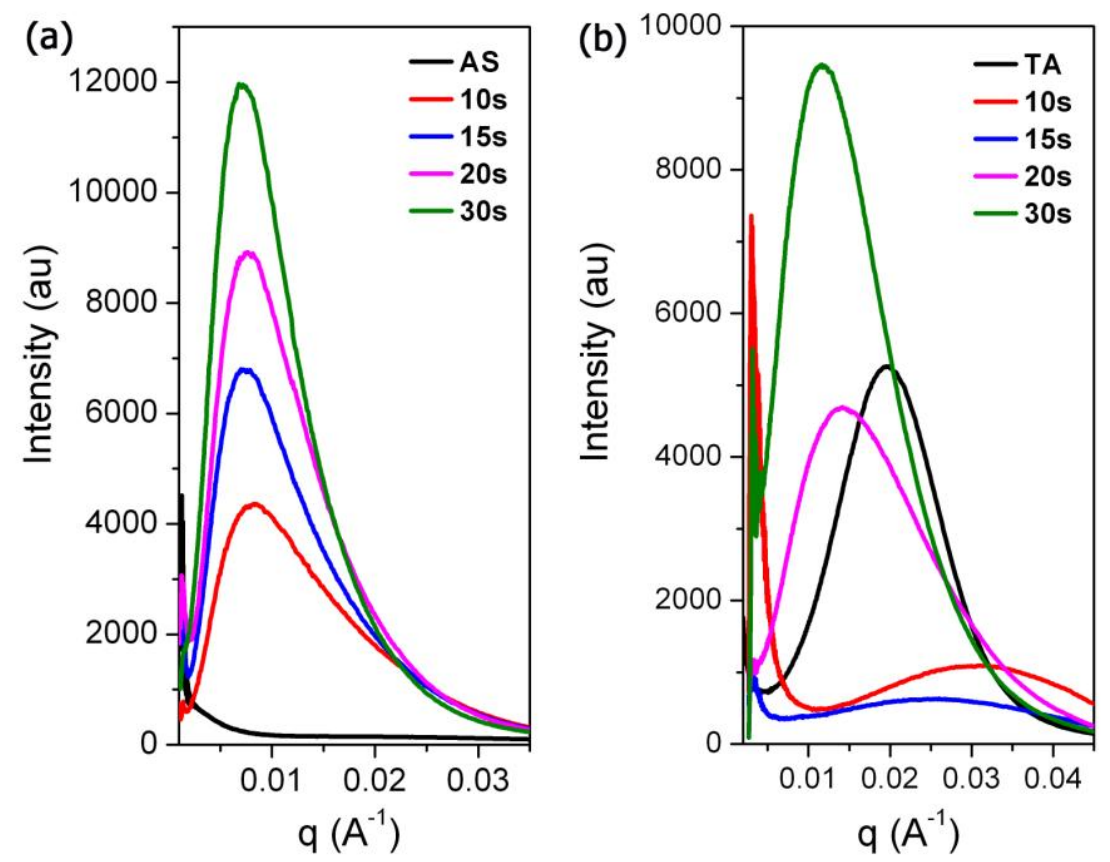

Figure 7. Resonant soft x-ray scattering for Py-SVA (a) and Py-TA-SVA (b) processed BHJ thin films.

The phase separation in BHJ blends was studied by resonant soft x-ray scattering (RSoXS). [39,40] Show in Figure 7a are the RSoXS results of Py-SVA experiment. It is clear that the as cast BHJ blends were well mixed, as evidenced by the absence of scattering over the scattering vector range $\left(0.005-0.03 \mathrm{~A}^{-1}(125-21 \mathrm{~nm})\right)$ investigate. With 10s SVA, a maximum in the scattering was seen at $\sim 0.008 \mathrm{~A}^{-1}$, corresponding to an average distance of $78.5 \mathrm{~nm}$ using Bragg's Law with the formula of $\mathrm{d}=2 \pi / \mathrm{q}$. Thus, in DPPZnPor-TBO:PCBM blends, the enhanced mobility imparted by the solvent led to phase separation. The appearance of a maximum in the scattering also indicates that the phase separation occurred by a spinodal phase separation mechanism. With subsequent SVA, the intensity of the reflection increased, indicating that the contrast between the phase separated domains increased, or that the concentration of the major component in both domains increased. In keeping with a spinodal mechanism, [41] the characteristic 
length scale remained constant. With respect to performance, 20s SVA sample gave the best PCE, and the $\mathrm{J}_{\mathrm{sc}}$ continued to increase up to this point, and then showed a slight decrease (from 20s to 30s). The device FF monotonically increased from 31.8 to 62.45 . Consequently, as phase separation proceeded, the purity of the domains increased, leading to an increase in the FF. The drop in $\mathrm{J}_{\mathrm{sc}}$ from 20-30s can be ascribed to a reduced localized PCBM depletion in the acceptor domains, which was similar to the morphological picture in PBDTPD thermal annealing case studies by McGhee and coworkers, $[42,43]$ since SVA would impart mobility to the BHJ thin film and lead to PCBM aggregations.

In Py-TA-SVA sample series, the 0s annealed thin film showed a very sharp reflection at $\sim 0.02 \mathrm{~A}^{-1}$, corresponding to a distance of $31.4 \mathrm{~nm}$. After 10s SVA, the reflection broadened substantially and shifted to $\sim 0.03 \mathrm{~A}^{-1}$, corresponding to a distance of $20.1 \mathrm{~nm}$. A PCE of $8.23 \%$ was seen for the corresponding device. 15s SVA led to a further reduction in the intensity of the scattering peak intensity, a broadening of the reflection to a shoulder at $\sim 0.026 \mathrm{~A}^{-1}$, corresponding to a distance of $24.1 \mathrm{~nm}$. Thus, the swelling of the BHJ thin films and dissolution of DPPZnPor-TBO crystals bring the system to an intermediate state of mixing as evidenced by the reduced scattering intensity. After 20s of SVA, a sharp phase separation at $0.014 \mathrm{~A}^{-1}(44.8 \mathrm{~nm})$, a new feature formed by liquid-liquid phase separation; and 30s SVA led to further enhancement of the scattering intensity at a similar position. Thus, the 20s to 30s SVA results in a phase purification similar to that seen in the Py-SVA experiment. However, the length scale of phase separation in this case is much smaller in comparison to the as cast-SVA sample (78.5 nm). Thus thermal annealing before SVA process has significant impact on the 
final morphology. The TA induced DPPZnPor-TBO crystals could look-in the system to reduce the length scale of phase separation. This most like achieved by intermolecular interactions in porphyrin molecules that form tight chains to restrain large scale of phase separation. The resulting solar cell devices showed better performances comparing to SVA on as-cast BHJ thin films.

\subsection{Structure-property relationship}
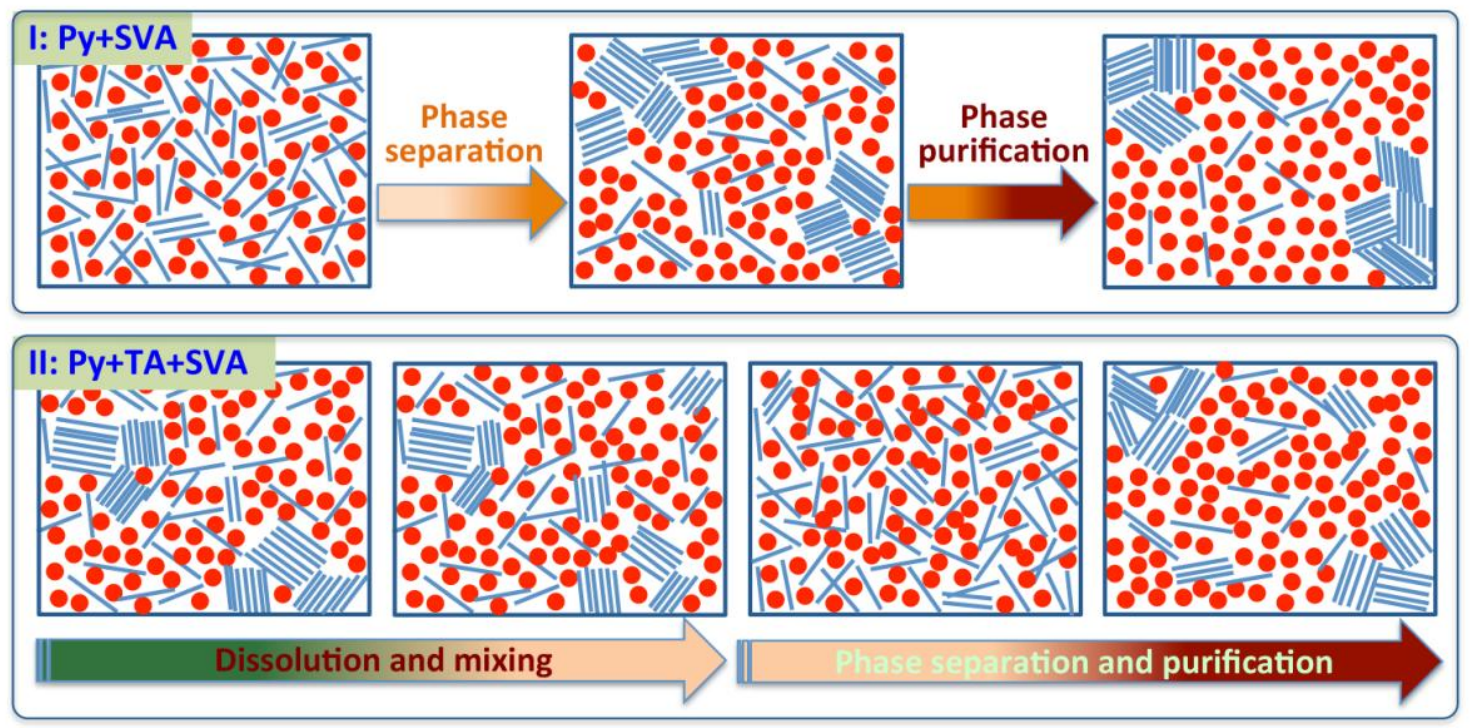

Scheme 2. Mechanism of phase separation in Py-SVA and Py-TA-SVA processed thin films.

The results presented above reveal the fundamental structure-property relationship of SVA in DPPZnPor-TBO:PCBM BHJ blends. The two sets of SVA samples show two different physical processes. A mechanistic understanding of these experiments is illustrated in Scheme 2. With the as-cast film, SVA leads to a rapid phase separation, with a slower crystallization of the donor molecule. The non-favorable segmental interactions, not crystallization or ordering, is the primary driving force for the phase separation, and does not significantly improve the PCE. As SVA continues, after 20s the 
phase separation increases, the donor crystallizes and device performance is optimized. Further annealing leads to higher phase purity, with a higher device $\mathrm{FF}$ but a lower $\mathrm{J}_{\mathrm{sc}}$, and the device PCE decreases. The Py-TA-SVA process was far more complicated than Py-SVA experiment. As shown in Scheme 2, there is evidence that two distinct subprocesses exist. The first is a dissolution, mixing and homogenization of the components. A phase separation then occurs from this uniformly mixed system occurs, followed by a phase purification, similar to Py-SVA case. We know that the DPPZnPor-TBO:PCBM mixture under a THF vapor will phase separate, as seen in the second step. The first step of crystal dissolution and mixing is a complicated process. The as-cast BHJ blends were well mixed, and subsequent thermal annealing at $100{ }^{\circ} \mathrm{C}$ could lead to DPPZnPor-TBO crystallization within the constraint of glassy PCBM. In the crystal growth front, there would be quite high stress, impinged by PCBM solids, and thus the length scale of phase separation is also small. When THF vapor get into thermal annealed BHJ thin film, the interfacial stress could be released by enhanced PCBM mobility, meanwhile stressed DPPZnPor-TBO crystals started to dissolve, leading to an intermediate mixing as seen from reduced crystallization and phase separation (from GIXD and RSoXS results). The best performing device under this condition was the early stage of SVA (10s) when there was still pronounced phase separation and certain amount of DPPZnPor-TBO crystallization. At this point, the internal stress is relieved as evidenced by the $\mathrm{H}$ aggregation peak position change. This process should be associated with PCBM aggregation, which drastically changes the global morphology of BHJ thin film (RSoXS results). It is interesting that in the processing, even when the phase separation reduced significantly, still quite good performance was seen (15s SVA, $8.24 \%$ PCE). This 
suggests there is some favorable intermolecular interactions in DPPZnPor-TBO, though the crystalline order is weak. In fact, the $\pi$ surface of DPPZnPor-TBO was quite large and the $\pi-\pi$ stacking, even in crystalline film, was not tight. Thus, the intermolecular holetransport in DPPZnPor-TBO can be achieved without well ordered $\pi-\pi$ stacking. This quite subtle interaction cannot be directly probed, which, however, had significant impact in thin film morphology and device performances. The subsequent SVA leads to a limited phase separation due to these subtle intermolecular interactions.

\section{Conclusions}

We have systematically studied the SVA of a high performance low band gap DPPZnPor-TBO:PCBM small molecule BHJ solar cell blends. In experiments, we see that molecular interaction and material crystallization are quite complicated factors that dictate the morphology and performances of BHJ thin films. When different thin film fabrication preparation protocols are used, the same solvent vapor annealing procedure can be of quite different physical nature and leads to markedly different morphologies. The time duration of SVA is a critical parameter that processing step that must be considered to optimize device performance. A power conversion efficiency of $8.48 \%$ was achieved, which is among the highest of deep absorption conjugated semiconductor solar cells.

\section{Acknowledgement}

K. Gao, W. Deng and L. Xiao contributed equally to this work. XBP and HBW was financially supported by the grants from International Science \& Technology Cooperation Program of China (2013DFG52740, 2010DFA52150), the Ministry of 
Science and Technology (2014CB643500), and the National Natural Science Foundation of China $(51473053,51073060,51225301,91333206)$. FL and TPR were supported by the U.S. Office of Naval Research under contract N00014-15-1-2244. Portions of this research were carried out at beamline 7.3.3 and 11.0.1.2 at the Advanced Light Source, Molecular Foundry, and National Center for Electron Micoscopy, Lawrence Berkeley National Laboratory, which was supported by the DOE, Office of Science, and Office of Basic Energy Sciences.

Received: ((will be filled in by the editorial staff))

Revised: ((will be filled in by the editorial staff))

Published online: ((will be filled in by the editorial staff))

\section{References:}

1 Y. Lin, J. Wang, Z. Zhang, H. Bai, Y. Li, D. Zhu and X. Zhan, Adv. Mater. 27 (2015) 1170-1174.

2 W. Zhao, D. Qian, S. Zhang, S. Li, O. Inganäs, F. Gao and J. Hou, Adv. Mater. 28 (2016) 4734-4739.

3 T. S. v. d. Poll, J. A. Love, T.-Q. Nguyen and G. C. Bazan, Adv. Mater. 24 (2012) 3646-3649.

4 B. Kan, Q. Zhang, M. Li, X. Wan, W. Ni, G. Long, Y. Wang, X. Yang, H. Feng and Y. Chen, J. Am. Chem. Soc. 136 (2014) 15529-15532.

5 Y. Sun, G. C. Welch, W. L. Leong, C. J. Takacs, G. C. Bazan and A. J. Heeger, Nat. Mater. 11 (2011) 44-48.

6 Q. Zhang, B. Kan, F. Liu, G. Long, X. Wan, X. Chen, Y. Zuo, W. Ni, H. Zhang, M. Li, Z. Hu, F. Huang, Y. Cao, Z. Liang, M. Zhang, T. P. Russell and Y. S. Chen, Nat. Photon. 9 (2015) 35-41.

7 Y. Lin, Y. Li and X. Zhan, Chem. Soc. Rev. 41 (2012) 4245-4272.

8 L. Gao, Z.-G. Zhang, H. Bin, L. Xue, Y. Yang, C. Wang, F. Liu, T. P. Russell and Y. Li, Adv. Mater. 37 (2016) 8288-8295

9 F. Liu, S. Ferdous, E. Schaible, A. Hexemer, M. Church, X. Ding, C. Wang and T. P. Russell, Adv. Mater. , 27 (2015) 886-891.

10 F. Liu, Y. Gu, X. Shen, S. Ferdous, H.-W. Wang and T. P. Russell, Prog. Polym. Sci. 38 (2013) 1990-2052.

11 B. Walker, A. Tamayo, D. T. Duong, X.-D. Dang, C. Kim, J. Granstrom and T.-Q. Nguyen, Adv. Energy Mater. 1 (2011) 221-229.

12 Y. Liu, X. Wan, F. Wang, J. A. Zhou, G. Long, J. Tian, J. You, Y. Yang and Y. Chen, Adv. Energy Mater. 1 (2011) 771-775.

13 C. Cui, X. Guo, J. Min, B. Guo, X. Cheng, M. Zhang, C. J. Brabec and Y. Li, Adv. Mater. 27 (2015) 74697475.

14 Y. Liu, X. Wan, F. Wang, J. A. Zhou, G. Long, J. Tian and Y. Chen, Adv. Mater. 23 (2011) 5387-5391.

15 B. Walker, A. B. Tamayo, X.-D. Dang, P. Zalar, J. H. Seo, A. Garcia, M. Tantiwiwat and T.-Q. Nguyen, Adv. Funct. Mater. 19 (2009) 3063-3069.

16 Z. Wang, X. Xu, Z. Li, K. Feng, K. Li, Y. Li and Q. Peng, Adv. Electron. Mater. 2 (2016) 1600061.

17 K. Wang, M. Azouz, M. Babics, F. Cruciani, T. Marszalek, Q. Saleem, W. Pisula and P. M. Beaujuge, Chem. Mater. 28 (2016) 5415-5425

18 M. Li, F. Liu, X. Wan, W. Ni, B. Kan, H. Feng, Q. Zhang, X. Yang, Y. Wang, Y. Zhang, Y.Shen, T. P. Russell and Y. Chen Adv. Mater. 27 (2015) 6296-6302.

19 J.-L. Wang, K.-K. Liu, J. Yan, Z. Wu, F. Liu, F. Xiao, Z.-F. Chang, H.-B. Wu, Y. Cao and T. P. Russell, J. Am. Chem. Soc. 138 (2016) 7687-7697

20 J. Min, X. Jiao, I. Ata, A. Osvet, T. Ameri, P. Bäuerle, H. Ade and C. J. Brabec, Adv. Energy Mater. 6 (2016) 1502579

21 K. Gao, J. Miao, L. Xiao, W. Deng, Y. Kan, T. Liang, C. Wang, F. Huang, J. Peng, Y. Cao, F. Liu, T. P. Russell, H. B. Wu and X. Peng, Adv. Mater. 28 (2016) 4727-4733.

22 K. Gao, L. Li, T. Lai, L. Xiao, Y. Huang, F. Huang, J. Peng, Y. Cao, F. Liu, T. P. Russell and X. Peng, J. Am. Chem. Soc. 137 (2015) 7282-7285.

23 V. Coropceanu, J. Cornil, D. A. da Silva Filho, Y. Olivier, R. Silbey and J.-L. Bredas, Chem. Rev. 107 (2007) 926-952.

24 P. W. M. Blom, V. D. Mihailetchi, L. J. A. Koster and D. E. Markov, Adv. Mater. 19 (2007) 1551-1566. 
25 M. M. Mandoc, W. Veurman, L. J. A. Koster, B. de Boer and P. W. M. Blom, Adv. Funct. Mater. 17 (2007) $2167-2173$.

26 J. Zhao, A. Swinnen, G. Van Assche, J. Manca, D. Vanderzande and B. V. Mele, J. Phys. Chem. B 113 (2009) $1587-1591$.

27 A. Foertig, J. Rauh, V. Dyakonov and C. Deibel, Phys. Rev. B 86 (2012) 115302.

28 R. A. Street, Phys. Rev. B 84 (2011) 075208.

29 R. C. I. MacKenzie, C. G. Shuttle, M. L. Chabinyc and J. Nelson, Adv. Energy Mater. 2 (2012) 662-669.

30 Z. Wang, Y. Zhang, J. Zhang, Z. Wei and W. Ma, Adv. Energy Mater. 6 (2016) 1502456

31 J. Zhang, Y. Zhang, J. Fang, K. Lu, Z. Wang, W. Ma and Z. Wei, J. Am. Chem. Soc. 137 (2015) 8176-8183.

32 M. E. Aydin, F. Yakuphanoglu, J.-H. Eom and D.-H. Hwang, Physica B: Condensed Matter. 387 (2007) 239244.

33 M. Meier, S. Karg and W. Riess, J. Appl. Phys. 82 (1997) 1961.

34 G. Garcia-Belmonte, A. Munar, E. M. Barea, J. Bisquert, I. Ugarte and R. Pacios, Org. Electron. 9 (2008) 847-851.

35 A. Hexemer, W. Bras, J. Glossinger, E. Schaible, E. Gann, R. Kirian, A. MacDowell, M. Church, B. Rude and H. Padmore, J. Phys.: Conf. Ser. 247 (2010) 012007.

36 M.-Y. Chiu, U.-S. Jeng, C.-H. Su, K. S. Liang and K.-H. Wei, Adv. Mater. 20 (2008) 2573-2578.

37 J. D. Zimmerman, X. Xiao, C. K. Renshaw, S. Wang, V. V. Diev, M. E. Thompson and S. R. Forrest, Nano Letters, 12 (2012) 4366-4371

38 G. Wei, R. R. Lunt, K. Sun, S. Wang, M. E. Thompson and S. R. Forrest, Nano Letters. 10 (2010) 3555-3559

39 F. Liu, M. A. Brady and C. Wang, Eur. Polym. J. (2016) http://dx.doi.org/10.1016/j.eurpolymj.2016.04.014.

40 E. Gann, A. T. Young, B. A. Collins, H. Yan, J. Nasiatka, H. A. Padmore, H. Ade, A. Hexemer and C.Wang, Rev. Sci. Instrum. 83 (2012) 045110.

41 J. W. Cahn, J. Chem. Phys. 42 (1965) 93.

42 J. A. Bartelt, Z. M. Beiley, E. T. Hoke, W. R. Mateker, J. D. Douglas, B. A. Collins, J. R. Tumbleston, K. R. Graham, A. Amassian, H. Ade, J. M. J. Frechet, M. F. Toney and M. D. Mcgehee, Adv. Energy Mater. 3 (2013) 364-371.

43 G. Wei, S. Wang, K. Sun, M. E. Thompson and S. R. Forrest, Adv. Energy Mater. 1 (2011) 184-187 
New Insight of Molecular Interaction, Crystallization and Phase Separation in Higher Performance Small Molecular Solar Cells Via Solvent Vapor Annealing Ke Gao ${ }^{a b \mathscr{l}}$ Wanyuan Deng, ${ }^{b \mathscr{l}}$ Liangang Xiao, ${ }^{b \mathscr{I}}$ Qin Hu, ${ }^{d}$ Yuanyuan Kan, ${ }^{b}$ Xuebin Chen, ${ }^{b}$ Cheng Wang, ${ }^{e}$ Fei Huang, ${ }^{b}$ Junbiao Peng, ${ }^{b}$ Hongbin Wu, ${ }^{b} *$ Xiaobin Peng ${ }^{b, *}$ Yong Cao, ${ }^{b}$ Thomas P. Russell, ${ }^{c, d}$ * and Feng Liu, ${ }^{a, d} *$

Graphical abstract

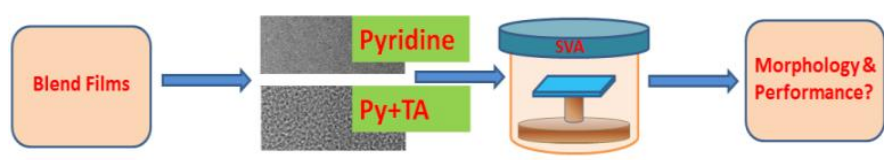

